\title{
Resonant Pumping of $d-d$ Crystal Field Electronic Transitions as a Mechanism of Ultrafast Optical Control of the Exchange Interactions in Iron Oxides
}

\author{
R. V. Mikhaylovskiy®, ${ }^{1,2, *}$ T. J. Huisman, ${ }^{1}$ V. A. Gavrichkov® ${ }^{3}$ S. I. Polukeev $\odot,{ }^{3}$ S. G. Ovchinnikov $\odot,{ }^{3}$ D. Afanasiev, ${ }^{1,4}$ \\ R. V. Pisarev ${ }^{5}{ }^{5}$ Th. Rasing, ${ }^{1}$ and A. V. Kimel ${ }^{1}$ \\ ${ }^{1}$ Radboud University, Institute for Molecules and Materials, Heyendaalseweg 135, 6525 AJ Nijmegen, The Netherlands \\ ${ }^{2}$ Department of Physics, Lancaster University, Bailrigg, Lancaster LA1 4YW, United Kingdom \\ ${ }^{3}$ Kirensky Institute of Physics, Federal Research Center KSC SB RAS, 660036 Krasnoyarsk, Russia \\ ${ }^{4}$ Kavli Institute of Nanoscience, Delft University of Technology, P.O. Box 5046, 2600 GA Delft, The Netherlands \\ ${ }^{5}$ Ioffe Physical-Technical Institute, Russian Academy of Sciences, 194021 St. Petersburg, Russia
}

(Received 26 November 2019; accepted 20 August 2020; published 9 October 2020; corrected 21 October 2020)

\begin{abstract}
The microscopic origin of ultrafast modification of the ratio between the symmetric $(J)$ and antisymmetric $(D)$ exchange interaction in antiferromagnetic iron oxides is revealed, using femtosecond laser excitation as a pump and terahertz emission spectroscopy as a probe. By tuning the photon energy of the laser pump pulse we show that the effect of light on the $D / J$ ratio in two archetypical iron oxides $\mathrm{FeBO}_{3}$ and $\mathrm{ErFeO}_{3}$ is maximized when the photon energy is in resonance with a spin and parity forbidden $d-d$ transition between the crystal-field split states of $\mathrm{Fe}^{3+}$ ions. The experimental findings are supported by a multielectron model, which accounts for the resonant absorption of photons by $\mathrm{Fe}^{3+}$ ions. Our results reveal the importance of the parity and spin-change forbidden, and therefore often underestimated, $d-d$ transitions in ultrafast optical control of magnetism.
\end{abstract}

DOI: 10.1103/PhysRevLett.125.157201

The short-range spin-spin exchange interaction that results in long-range magnetic order is one of the important demonstrations of the quantum nature of matter. Remarkably, the strength of the exchange interaction in magnetically ordered materials expressed in terms of effective magnetic fields can reach $1000 \mathrm{~T}$ in which the spin precession period is typically shorter than a picosecond. Controlling the exchange interaction by subpicosecond laser pulses is thus a very appealing approach to search for a new scenario of the fastest possible control of magnetism [1].

The symmetric part of the exchange energy $W_{\mathrm{ex}}$ between two magnetic sublattices $\mathbf{S}_{1}$ and $\mathbf{S}_{2} W_{\mathrm{ex}}=J \mathbf{S}_{1} \mathbf{S}_{2}$ is responsible for the very existence of long-range magnetic order. During recent years, the modulation of the symmetric exchange interaction by femtosecond laser pulses has been a subject of experimental and theoretical studies [2-19]. Its antisymmetric counterpart, the relativistic DzyaloshinskiiMoriya energy $W_{D}=\mathbf{D} \times\left[\mathbf{S}_{1} \times \mathbf{S}_{2}\right]$, contributes to the emergence of weak ferromagnetism [20], multiferroicity [21], and magnetic skyrmions [22]. The possibility of ultrafast modification of the ratio between $J$ and $D$ has been reported for various transition metal oxides and motivated several theoretical proposals to manipulate magnetic textures through optical control [23-28]. However, presently available theoretical models fail to describe the change of exchange in realistic materials, hence there is no information on which optical transitions one must pump to change $D / J$ efficiently. In this Letter we experimentally reveal that the weak, and thus often overlooked, $d-d$ transitions are responsible for the efficient modification of the $D / J$ exchange ratio. We also suggest a multiorbital theory that can explain the effect.

To observe the optical modification of $J$ and/or $D$, one can use the fact that in a broad class of antiferromagnetic iron oxides (iron borate $\mathrm{FeBO}_{3}$, hematite $\alpha-\mathrm{Fe}_{2} \mathrm{O}_{3}$, and orthoferrites $R \mathrm{FeO}_{3}$, with $R$ being a rare-earth element) the ratio $D / J$ defines the canting angle of the two magnetic sublattices of $\mathrm{Fe}^{3+}$ ions. Therefore, in these materials an ultrafast change of the exchange constants $J$ and/or $D$ results in coherent spin motion that can be reliably separated from the heat-driven and other incoherent dynamics. Particularly, perturbation of $J$ and/or $D$ triggers the quasiantiferromagnetic (q-AFM) mode of antiferromagnetic resonance [29] involving periodic oscillation of the canting angle at a $\mathrm{THz}$ frequency. The spin motion acts as ac magnetic dipole emitting coherent $\mathrm{THz}$ electromagnetic waves, which were measured experimentally. The excitation of the antiferromagnetic resonance by light can be seen as an impulsive stimulated Raman process, known as inverse magnetic refraction. Microscopically, this effect involves the change of the $J / D$ ratio [29].

The optical absorption of the iron oxides is defined by charge-transfer electric dipole transitions between the oxygen $p$ orbitals and the $d$ orbitals of the $\mathrm{Fe}^{3+}$ ions and $d-d$ transitions of the single $\mathrm{Fe}^{3+}$ ion (see Fig. 1 and Refs. [30-32]). The virtual hopping of the electrons between the $\mathrm{Fe}^{3+}$ and $\mathrm{O}^{2-}$ ions gives rise to superexchange 


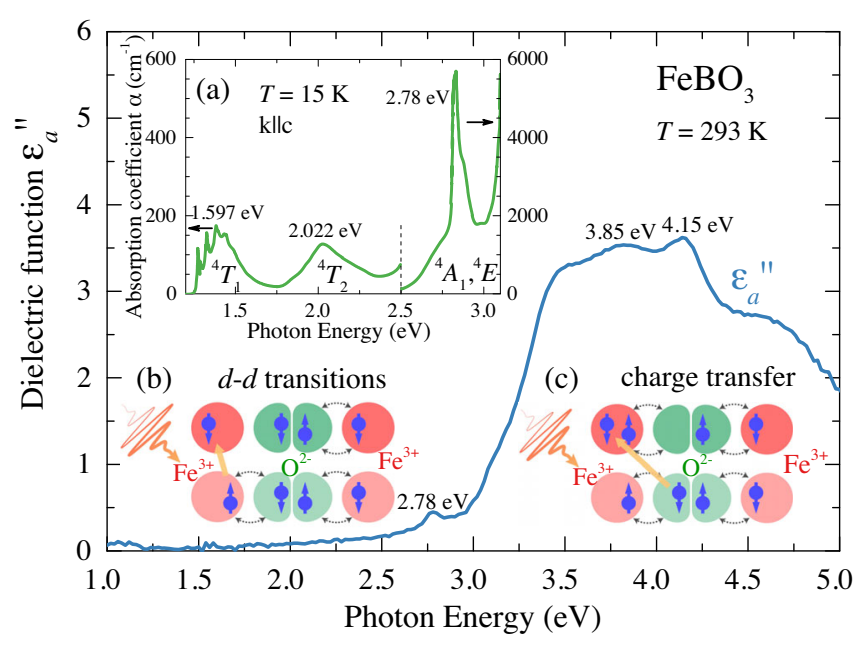

FIG. 1. Main panel: The imaginary part of the $\mathrm{FeBO}_{3}$ dielectric function as a function of photon energy measured using a spectroscopic ellipsometer. The response is dominated by strong charge transfer transitions above $3 \mathrm{eV}$. Insets: (a) Absorption coefficient of antiferromagnetic iron oxide $\mathrm{FeBO}_{3}$. Absorption was measured for light propagating along the optical axis. Absorption bands due to the $d-d$ transitions from the ${ }^{6} A_{1}$ ground state to the ${ }^{4} T_{1},{ }^{4} T_{2},{ }^{4} A_{1},{ }^{4} E$ excited states are indicated. (b). Modulation of the superexchange interaction due to the pumping of the $d-d$ transitions involving a spin flip from $S=$ $5 / 2$ in the ground state to $S=3 / 2$ in the excited states. (c). Schematics showing the modulation of the superexchange interaction by a laser induced charge transfer.

interaction resulting in the antiferromagnetic ordering $(J>0)$. Therefore, it is natural to assume that the laser pulse excites the charge-transfer transitions, thereby modifying the hopping and consequently the exchange coupling between the neighboring $\mathrm{Fe}^{3+}$ ions. This scenario is illustrated in Fig. 1(c) and has been discussed earlier for manganites [33] and ferromagnetic or antiferromagnetic heterostructures $[34,35]$.

The weak and broad $d-d$ absorption bands arise due to the interorbital transitions between the $3 d$ states split by the crystal field (see Fig. 1 for $\mathrm{FeBO}_{3}$ and Refs. [30,31] for other iron oxides). These transitions are forbidden in the electric-dipole approximation. However, they become partially allowed due to mixing of $p-d$ atomic states of opposite parity by phonons and/or due to the inversion symmetry breaking at the position of the magnetic ion. Moreover, these electronic excitations between the $3 d^{5}$ states of the $\mathrm{Fe}^{3+}$ ion require a spin change from $S=5 / 2$ to $S=3 / 2$ that is also forbidden for optical transitions in the electric-dipole approximation. However, this restriction is removed by accounting for the spin-orbit coupling. A femtosecond optical pulse can excite these transitions resonantly and drive electrons into a new orbital configuration with a different spin value, thereby perturbing the superexchange [Fig. 1(b)]. Even though most pump-probe experiments use pump pulses with a photon energy of
$1.55 \mathrm{eV}$ very close to the $d-d$ absorption bands in iron oxides, the interorbital transitions in magnetic cations in ultrafast light-spin interactions has so far got very limited attention [36-38] and their role in the control of the $D / J$ exchange ratio still remains unclear.

Spectral measurements with wavelength-tuneable laser pulses can provide an efficient way for unveiling the microscopic mechanisms responsible for the modification of the exchange interaction. To establish a spectroscopic correlation between the observed effects and the absorption bands, we employed $\mathrm{THz}$ emission spectroscopy [39], with tunable photon energy of the pump laser pulse [40]. The measured $\mathrm{THz}$ signal is directly related to the magnetization dynamics by linear Maxwell equations [41]. The samples were brought into a single domain state by an in-plane bias magnetic field of $\sim 0.1 \mathrm{~T}$.

Initially we studied a rhombohedral calcite-type single crystal of iron borate $\mathrm{FeBO}_{3}$. The $370 \mu$ m thick sample was cut perpendicularly to the $z$ axis, i.e., with the antiferromagnetic vector and weak ferromagnetic moment in the basal $x y$ plane. Figure 2(a) shows the time traces of the electric field emitted from the photoexcited sample for different pump pulse photon energies. In order to maximize the detected signal, the measurements were done at the low temperature of $10 \mathrm{~K}$. As one can see, the signals consist of quasimonochromatic oscillations at a frequency of about $450 \mathrm{GHz}$, matching the frequency of the q-AFM mode of $\mathrm{FeBO}_{3}$. The observed waveforms do not depend either on the polarization of the pump light, or on the crystal orientation, while their sign does change when changing the polarity of the applied magnetic field. The signals have all the properties attesting the excitation of the q-AFM by modulation of the superexchange interaction [29]. (a)

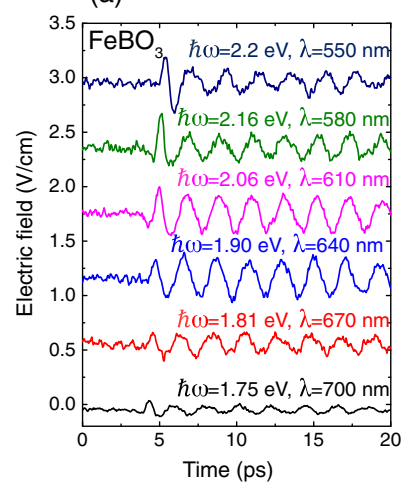

(b)

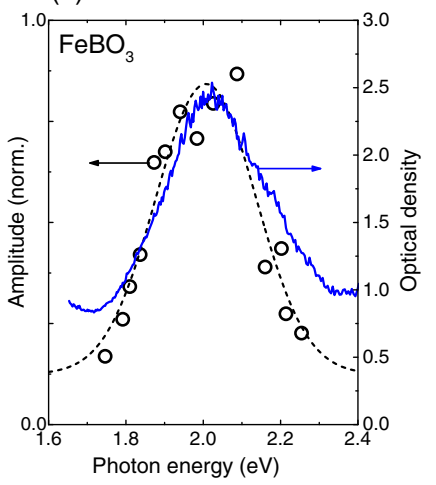

FIG. 2. (a). Waveforms of the THz electric field emitted from $\mathrm{FeBO}_{3}$ excited by femtosecond pulses of different photon energy (wavelength). The slight change in the signal delay is due to the inequality of the optical paths for different wavelengths. (b). Amplitude of the $\mathrm{THz}$ waveforms as a function of the pump photon energy (open circles) shown together with the measured optical density of the same sample (solid line). The amplitude is normalized with respect to the pump power, which varied for different wavelengths. Dashed line is a guide to the eye. 
By fitting the experimental data with decaying sinusoidal functions, we retrieved the amplitude of the q-AFM mode, which is plotted in Fig. 2(b) as a function of the pump photon energy. The amplitude shows a clear resonant behavior in the vicinity of the ${ }^{6} A_{1} \rightarrow{ }^{4} T_{2}$ transition, with a central energy at $\sim 2 \mathrm{eV}$ [see Fig. 1(a)]. To further support this observation, we measured the optical transmission as a function of photon energy for this particular sample. The resulting optical density perfectly matches the dependence of the q-AFM amplitude [see Fig. 2(b)], confirming that the optical excitation of the q-AFM mode and hence the modulation of the $D / J$ exchange ratio is due to the resonant pumping of the ${ }^{6} A_{1} \rightarrow{ }^{4} T_{2}$ transition.

To test whether the observed resonant behavior is present in other iron oxides, we repeated similar $\mathrm{THz}$ emission measurements on $\mathrm{ErFeO}_{3}$, belonging to the orthorhombic crystal family of rare-earth orthoferrites. In this material we also observed $\mathrm{THz}$ emission corresponding to the q-AFM mode [29]. As an example, Fig. 3 shows the photon energy dependence of the amplitude of the q-AFM mode of the $\mathrm{ErFeO}_{3}$ sample. The optical density of the sample is also shown in Fig. 3. Very similar to $\mathrm{FeBO}_{3}$ the amplitude peaks at the photon energy corresponding to the ${ }^{6} A_{1} \rightarrow{ }^{4} T_{2}$ absorption band. At the same time, there is no clear evidence for contribution of $f-f$ transitions between $\mathrm{Er}^{3+}$ localized states (seen as narrow peaks just below $1.9 \mathrm{eV}$ in Fig. 3) to the perturbation of the $D / J$ ratio. However, THz emission efficiency seems to be centered at a slightly higher photon energy compared to the $d-d$ absorption band. It may indicate some influence of the $f-f$

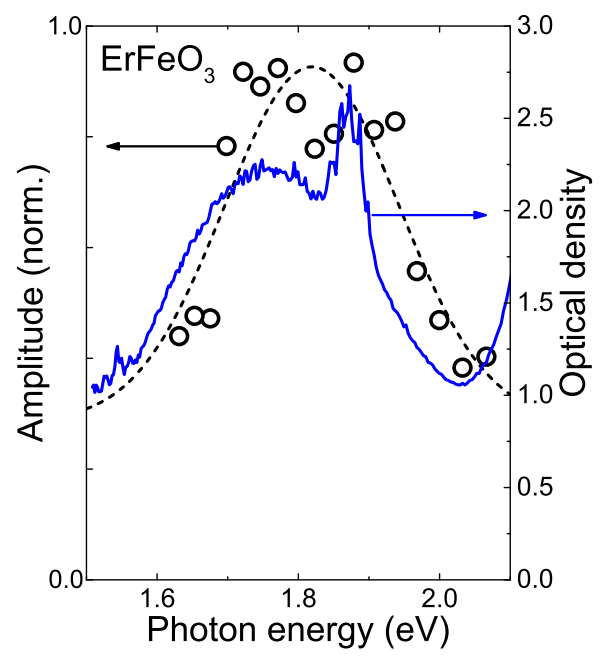

FIG. 3. Amplitude of the $\mathrm{THz}$ waveforms as a function of the pump photon energy (open circles) shown along with the measured optical density of the $\mathrm{ErFeO}_{3}$ sample (solid line). The amplitude is normalized as in Fig. 2. Dashed line is a guide to the eye. The narrow feature at $\sim 1.87 \mathrm{eV}$ is due to $f-f$ transitions in $\mathrm{Er}^{3+}$ ions. This measurement was performed at room temperature. transitions or it may also be due to the velocity mismatch between $\mathrm{THz}$ and optical waves at the different frequencies.

Using a $\beta$-barium borate (BBO) single crystal the photon energy of the fundamental laser pulse at $1.55 \mathrm{eV}$ was doubled to $3.1 \mathrm{eV}$ in order to pump the samples in the region of the strong absorption close to their charge-transfer gap (Fig. 1). However, we observed no THz emission from all samples in this case. Although this result stands against a charge-transfer based mechanism of the superexchange modulation [Fig. 1(c)], one has to bear in mind that due to the strong absorption of more than $10^{3} \mathrm{~cm}^{-1}$ at $3.1 \mathrm{eV}$, the penetration depth of the laser pulse is only $\sim 1 \mu \mathrm{m}$, which is much less than that in the transparency region $(\sim 70 \mu \mathrm{m})$. Therefore, the $\mathrm{THz}$ signal is emitted from a significantly thinner part of the sample than in the $1.7-2.3 \mathrm{eV}$ range. Most probably it falls below the noise level and thus requires more detailed study. Nonetheless, we can confidently state that the pumping of $d-d$ transitions positioned below the charge transfer transitions dominates in the optical modulation of the $D / J$ exchange ratio in the iron oxides.

The existing models for nonequilibrium exchange [1] neglect the possibility of laser-induced spin flips due to magnetic dipole transitions or nonlocal optical transitions associated with the generation of exciton-magnon pairs at the neighboring lattice sites [42]. Here we theoretically discuss how the incorporation of the excited states of the magnetic cation can result in a change of the superexchange interaction under optical pumping. We employ the formalism recently developed in Ref. [43]. We consider a rareearth free case of $\mathrm{FeBO}_{3}$. In its ground state a nonzero value of the spin canting angle $\varphi_{0} \approx 0.95^{\circ}$ is observed, which can be explained as a result of Dzyaloshinskii-Moriya interaction [44]. Under optical pumping, the ${ }^{4} T_{2}$ triplet excited states with spin $S=\frac{3}{2}$ are populated. The conclusion of the multielectron approach [43] is that the resonant occupation of some excited states of the $\mathrm{Fe}^{3+}$ ions under optical pumping may change the value and even the sign of the superexchange interaction between the excited ion and a neighboring ion in the ground state. For the ${ }^{4} T_{2}$ excited term of the $\mathrm{Fe}^{3+}$ ion a ferromagnetic (FM) type of exchange has been found [45].

To clarify the physics of the complicated multielectron approach [43] we illustrate the optical modulation of the superexchange interaction in Fig. 4. At equilibrium the superexchange arises from electron hopping between two $\mathrm{Fe}^{3+}$ ions in the ground state with $S=5 / 2$ via an $\mathrm{O}^{2-}$ ion (see the lower part of Fig. 4). One spin-up ( $\uparrow$ ) electron from the left cation virtually hops to oxygen forming a $\uparrow \downarrow$ pair and back with the same spin projection. Another oxygen spin-up electron also virtually hops to the right cation to the spin-down $(\downarrow)$ electron and back. Such mechanism favors the antiferromagnetic (AFM) ordering between the neighboring $\mathrm{Fe}^{3+}$ ions. After a photoinduced $d-d$ transition (upper part of Fig. 4) one of the electrons in the excited ion 


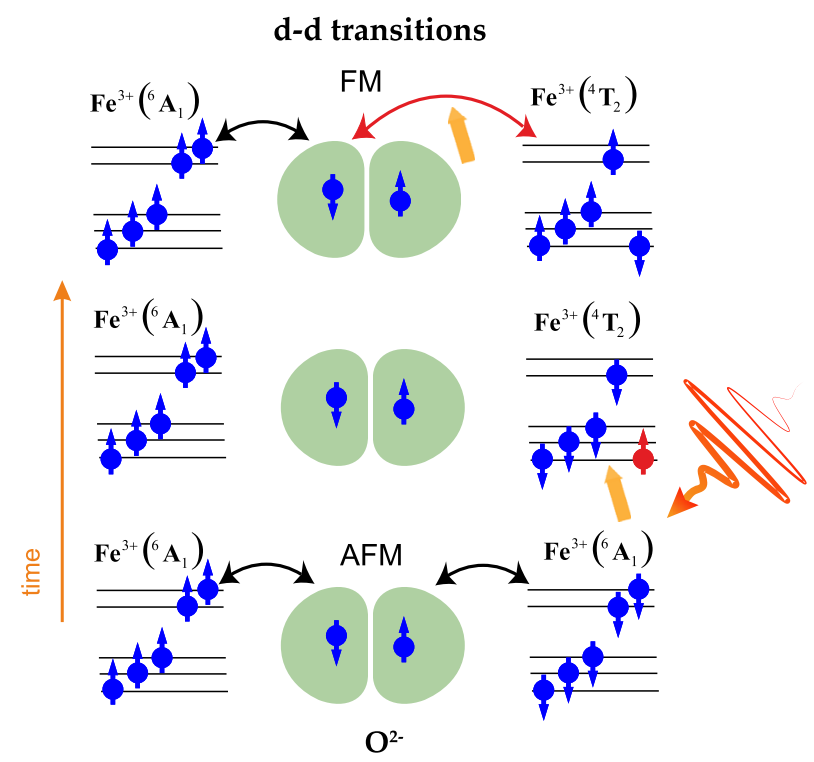

FIG. 4. Illustration of the photoinduced modulation of the superexchange interaction in an iron oxide. In the ground state (lower panel) the hopping results in the antiferromagnetic alignment of spins. The optical excitation flips one spin in an iron ion (red one, middle panel) and as a result in the excited state (upper panel) the interaction becomes ferromagnetic and more spins change their orientation.

is antiparallel to the others and the spin of the excited term becomes $3 / 2$. The virtual hopping of the spin-up electron from the left cation in the ground state to oxygen and back is the same as it was before the laser excitation, while for the right excited cation the virtual hopping of the second oxygen spin-down electron and back is possible for the spin-up cation, where four electrons of the excited ${ }^{4} T_{2}$ term remain parallel to the spin of the left cation, so their interaction becomes ferromagnetic (FM). We should remark that this picture is just a cartoon of the complicated calculation $[43,46]$ where the effective spin Hamiltonian including both ions in the ground and excited states is obtained by means of a perturbation theory.

Besides the qualitative picture shown in Fig. 4, direct calculations [43] lead to an AFM superexchange in the ground state, and to a FM interaction $J_{i j}^{\text {ex }}$ for $\mathrm{Fe}^{3+}$ ions under optical pumping at $A$ and $B$ absorption lines of a set of $A\left({ }^{4} T_{1}\right), B\left({ }^{4} T_{2}\right)$, and $C\left({ }^{4} A_{1},{ }^{4} E\right)$ optical $d-d$ excitations [45]. The optically induced FM contributions to the superexchange $J_{i j}^{\text {ex }}$ dominate due to the largest overlap of $2 p$ oxygen orbitals with excited $\mathrm{Fe}^{3+}\left({ }^{4} T_{2}\right)$ ions. When excited in the $C$ line, the FM contribution of the interaction $J_{i j}^{\text {ex }}$ vanishes due to a sharp drop in the overlapping for the excited $\mathrm{Fe}^{3+}\left({ }^{4} A_{1},{ }^{4} E\right)$ ions. The AFM state of $\mathrm{FeBO}_{3}$ is maintained at equilibrium at a low concentration of excited ion pairs $\mathrm{Fe}^{3+}\left({ }^{4} T_{2}\right)-\mathrm{Fe}^{3+}\left({ }^{6} A_{1}\right)$ with $\mathrm{FM}$ exchange. We assume that the optical transition occurs instantaneously, and the lifetime of the excited $\mathrm{Fe}^{3+}$ ion exceeds the characteristic time of the change in the superexchange interaction $h / W \sim 10^{-15} \mathrm{~s}$, where $W$ is the bandwidth.

The spin-orbit interaction appears already in the first order of perturbation theory and results in a small change in the spin-canting angle. However, this last contribution is not important in the model under discussion, because the main effect is the rearrangement of spins of the two AFM sublattices shown in Fig. 5. The lower part of Fig. 5 shows four spins in the ground state, two $\mathbf{S}_{1}$ from the $A$ sublattice and two $\mathbf{S}_{2}$ from the $B$ sublattice. The excitation of the $A$ sublattice ion is shown in the left part and that of the $B$ ion in the right part. There are two major effects of the $d-d$ excitations that should be taken into account. The first one is the spin change from $S=5 / 2$ to $S=3 / 2$, shown by dotted lines in the upper part of Fig. 5. The second effect is the excited spin rotation with the total spin $\mathbf{S}$ oriented left or right depending on which cation has been excited. It is evident that both sublattices are excited similarly, so the total magnetization is not changed. Nevertheless, the localized $d-d$ excitation forms the excited total magnetic moment $\mathbf{S}$. Therefore, the spin canting angle $\varphi_{\mathrm{ex}}$ at the optically excited $\mathrm{Fe}^{3+}\left({ }^{4} T_{2}\right)$ state is modified as determined by the changed values of the superexchange and spin-orbit interactions:

$$
\varphi_{\mathrm{ex}}=\left(\pi-\frac{D_{\mathrm{ex}}}{J_{i j}^{\mathrm{ex}}}\right)
$$

These processes are illustrated in Fig. 5. The phase shift $\sim \pi$ arises due to a spin flip at the optically excited $\mathrm{Fe}^{3+}\left({ }^{4} T_{2}\right)$ center in one of the magnetic sublattices under the action of the optically induced FM $J_{i j}^{\mathrm{ex}}>0$ superexchange interaction.

The most remarkable result of the optical response in $\mathrm{FeBO}_{3}$ under resonant pumping of the ${ }^{6} A_{1} \leftrightarrow{ }^{4} T_{2}$ transition is the sign change in the superexchange interaction from

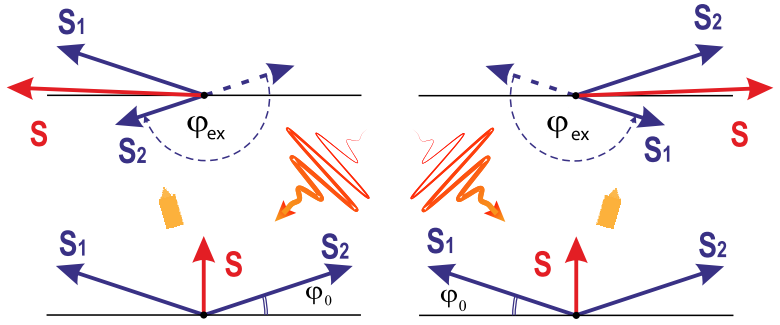

FIG. 5. Change of the relative orientation of the magnetic moments in an exchange-coupled pair of $\mathrm{Fe}^{3+}$ ions due to the sign change of the superexchange interaction from $\operatorname{AFM}\left(J_{i j}<0\right.$, lower part) to FM ( $J_{i j}^{\mathrm{ex}}>0$, upper part) induced by the laser pulse. The lower part shows the relative orientation of the spin moments $\mathbf{S}_{1}$ and $\mathbf{S}_{2}$ of the two ions in the ground state. The upper part shows the relative orientation of $\mathbf{S}_{1}$ and $\mathbf{S}_{2}$ of the two ions in which one is in the excited state. Left part of the figure corresponds to optical $d-d$ transitions in the magnetic sublattice $A$, where $S_{1}=5 / 2, S_{2}=3 / 2$, and the right part corresponds to $d-d$ transitions in the sublattice $B$, where $S_{1}=3 / 2, S_{2}=5 / 2$. 
AFM $J_{i j}<0$ to FM $J_{i j}^{\text {ex }}>0$. Because the optical absorption in both sublattices of the AFM material is identical, the total magnetic moment under optical pumping is still close to zero and no macroscopic FM ordering arises (Fig. 5). Evidently, in the linear regime the concentration $x$ of optically excited centers is proportional to the intensity of the optical pump, which should not be too large to avoid dielectric breakdown. Using the absorption coefficient of $\mathrm{FeBO}_{3}$ (Fig. 1), the size of its unit cell [47], the pump fluence, and the excitation volume, we may estimate that in our experiment $x \leq 10^{-3}$. This number is in line with our estimation of the modulation of the ratio $D / J \geq 10^{-4}$ [29].

In summary, we have shown that optical control of the exchange interaction in iron oxides can be achieved by resonant excitation of the $d-d$ crystal field transitions in magnetic $\mathrm{Fe}^{3+}$ ions, involving a spin change $\Delta S=1$. Our finding demonstrates another alternative to the currently used theoretical approaches based on the Hubbard model for the description of ultrafast light-spin interactions. It reveals novel opportunities for resonant optical control of the exchange interaction and thus opens up new perspectives for experimental and theoretical research in the field of ultrafast magnetism.

We thank A. Toonen and S. Semin for technical assistance. The work at Radboud University was supported by de Nederlandse Organisatie voor Wetenschappelijk Onderzoek (NWO) and the European Research Council ERC Grant Agreement No. 339813 (Exchange). R. V. M. thanks ERC, Grant Agreement No. 852050 (MAGSHAKE). V. A. G., S. I. P., and S. G. O. are thankful to the Russian Science Foundation for the financial support under the Grant No. 18-12-00022. The theoretical part of this research was performed by V.A. G., S.I.P., and S. G. O. under the RSF Grant No. 18-12-00022. The contribution of R.V.P. into the experimental part was supported by the RSF Grant No. 16-12-10456.

*r.mikhaylovskiy@lancaster.ac.uk

[1] J. H. Mentink, Manipulating magnetism by ultrafast control of the exchange interaction, J. Phys. Condens. Matter 29, 453001 (2017).

[2] H.-S. Rhie, H. Dürr, and W. Eberhardt, Femtosecond Electron and Spin Dynamics in Ni/W(110) Films, Phys. Rev. Lett. 90, 247201 (2003).

[3] A. Melnikov, I. Radu, U. Bovensiepen, O. Krupin, K. Starke, E. Matthias, and M. Wolf, Coherent Optical Phonons and Parametrically Coupled Magnons Induced by Femtosecond Laser Excitation of the Gd (0001) Surface, Phys. Rev. Lett. 91, 227403 (2003).

[4] J. Wang, I. Cotoros, K. Dani, X. Liu, J. Furdyna, and D. Chemla, Ultrafast Enhancement of Ferromagnetism via Photoexcited Holes in GaMnAs, Phys. Rev. Lett. 98, 217401 (2007).

[5] S. Wall, D. Prabhakaran, A.T. Boothroyd, and A. Cavalleri, Ultrafast Coupling between Light, Coherent
Lattice Vibrations, and the Magnetic Structure of Semicovalent $\mathrm{LaMnO}_{3}$, Phys. Rev. Lett. 103, 097402 (2009).

[6] M. Först, R. I. Tobey, S. Wall, H. Bromberger, V. Khanna et al., Driving magnetic order in a manganite by ultrafast lattice excitation, Phys. Rev. B 84, 241104(R) (2011).

[7] R. Carley, K. Döbrich, B. Frietsch, C. Gahl, M. Teichmann, O. Schwarzkopf, P. Wernet, and M. Weinelt, Femtosecond Laser Excitation Drives Ferromagnetic Gadolinium out of Magnetic Equilibrium, Phys. Rev. Lett. 109, 057401 (2012).

[8] T. Li, A. Patz, L. Mouchliadis, J. Yan, T. A. Lograsso, I. E. Perakis, and J. Wang Femtosecond switching of magnetism via strongly correlated spin-charge quantum excitations, Nature (London) 496, 69 (2013).

[9] R. R. Subkhangulov, A. B. Henriques, P. H. O. Rappl, E. Abramof, Th. Rasing, and A. V. Kimel All-optical manipulation and probing of the d-f exchange interaction in EuTe, Sci. Rep. 4, 4368 (2014).

[10] J. H. Mentink and M. Eckstein, Ultrafast Quenching of the Exchange Interaction in a Mott Insulator, Phys. Rev. Lett. 113, 057201 (2014).

[11] M. Matsubara, A. Schroer, A. Schmehl, A. Melville, C. Becher, M. Trujillo-Martinez, D. G. Schlom, J. Mannhart, J. Kroha, and M. Fiebig Ultrafast optical tuning of ferromagnetism via the carrier density, Nat. Commun. 6, 6724 (2015).

[12] J. H. Mentink, K. Balzer, and M. Eckstein, Ultrafast and reversible control of the exchange interaction in Mott insulators, Nat. Commun. 6, 6708 (2015).

[13] B. Frietsch, J. Bowlan, R. Carley, M. Teichmann, S. Wienholdt, D. Hinzke, U. Nowak, K. Carva, P. M. Oppeneer, and M. Weinelt, Disparate ultrafast dynamics of itinerant and localized magnetic moments in gadolinium metal, Nat. Commun. 6, 8262 (2015).

[14] J. O. Johansson, J.-W. Kim, E. Allwright, D. M. Rogers, N. Robertson, and J.-Y. Bigot, Directly probing spin dynamics in a molecular magnet with femtosecond time-resolution, Chem. Sci. 7, 7061 (2016).

[15] S. Eich et al. Band structure evolution during the ultrafast ferromagnetic-paramagnetic phase transition in cobalt, Sci. Adv. 3, e1602094 (2017).

[16] $\mathrm{Ph}$. Tengdin et al. Critical behavior within 20 fs drives the out-of-equilibrium laser-induced magnetic phase transition in nickel, Sci. Adv. 4, eaap9744 (2018).

[17] S. F. Maehrlein et al. Dissecting spin-phonon equilibration in ferrimagnetic insulators by ultrafast lattice excitation, Sci. Adv. 4, eaar5164 (2018).

[18] D. Afanasiev et al., Ultrafast Spin Dynamics in Photodoped Spin-Orbit Mott Insulator $\mathrm{Sr}_{2} \mathrm{IrO}_{4}$, Phys. Rev. X 9, 021020 (2019).

[19] A. Ron et al. Ultrafast enhancement of ferromagnetic spin exchange induced by ligand-to-metal charge transfer, arXiv: 1910.06376

[20] I. Dzyaloshinsky, A thermodynamic theory of "weak" ferromagnetism of antiferromagnetics, J. Phys. Chem. Solids 4, 241 (1958).

[21] I. A. Sergienko and E. Dagotto, Role of the DzyaloshinskiiMoriya interaction in multiferroic perovskites, Phys. Rev. B 73, 094434 (2006).

[22] U. K. Rößler, A. N. Bogdanov, and C. Pfleiderer, Spontaneous skyrmion ground states in magnetic metals, Nature (London) 442, 797 (2006). 
[23] C. Dutreix, E. A. Stepanov, and M. I. Katsnelson, Laserinduced topological transitions in phosphorene with inversion symmetry, Phys. Rev. B 93, 241404(R) (2016).

[24] E. A. Stepanov, C. Dutreix, and M. I. Katsnelson, Dynamical, and Reversible Control of Topological Spin Textures, Phys. Rev. Lett. 118, 157201 (2017).

[25] H. Fujita and M. Sato, Ultrafast generation of skyrmionic defects with vortex beams: Printing laser profiles on magnets, Phys. Rev. B 95, 054421 (2017).

[26] D. Yudin, D. R. Gulevich, and M. Titov, Light-Induced Anisotropic Skyrmion and Stripe Phases in a Rashba Ferromagnet, Phys. Rev. Lett. 119, 147202 (2017).

[27] T. H. Kim, P. Grünberg, S. H. Han, and B. K. Cho, Precessional switching of antiferromagnets by electric field induced Dzyaloshinskii-Moriya torque, Phys. Rev. B 97, 184427 (2018).

[28] F. Freimuth, S. Blügel, and Y. Mokrousov, Spin-orbit torques and tunable Dzyaloshinskii-Moriya interaction in Co/Cu/Co trilayers, Phys. Rev. B 98, 024419 (2018).

[29] R. V. Mikhaylovskiy et al. Ultrafast optical modification of exchange interactions in iron oxides, Nat. Commun. 6, 8190 (2015).

[30] D. L. Wood, J. P. Remeika, and E. D. Kolb, Optical spectra of rare earth orthoferrites, J. Appl. Phys. 41, 5315 (1970).

[31] A. M. Kalashnikova, V. V. Pavlov, R. V. Pisarev, L. N. Bezmaternykh, M. Bayer, and Th. Rasing, Linear and nonlinear optical spectroscopy of gadolinium iron borate $\mathrm{GdFe}_{3}\left(\mathrm{BO}_{3}\right)_{4}$, JETP Lett. 80, 293 (2004).

[32] R. V. Pisarev, A. S. Moskvin, A. M. Kalashnikova, and Th. Rasing, Charge transfer transitions in multiferroic $\mathrm{BiFeO}_{3}$ and related ferrite insulators, Phys. Rev. B 79, 235128 (2009).

[33] H. B. Zhao, D. Talbayev, X. Ma, Y. H. Ren, A. Venimadhav, Qi Li, and G. Lüpke, Coherent Spin Precession via Photoinduced Antiferromagnetic Interactions in $\mathrm{La}_{0.67} \mathrm{Ca}_{0.33} \mathrm{MnO}_{3}$, Phys. Rev. Lett. 107, 207205 (2011).

[34] X. Ma, F. Fang, Q. Li, J. Zhu, Y. Yang, Y.Z. Wu, H. B. Zhao, and G. Lüpke Ultrafast spin exchange-coupling torque via photo-excited charge-transfer processes, Nat. Commun. 6, 8800 (2015).

[35] Z. Zheng et al. Ultrafast modulation of exchange-coupling induced anisotropy in $\mathrm{Fe} / \mathrm{CoO}$ by laser induced charge transfer, Appl. Phys. Lett. 110, 172401 (2017).

[36] R. Iida, T. Satoh, T. Shimura, K. Kuroda, B. A. Ivanov, Y. Tokunaga, and Y. Tokura, Spectral dependence of photoinduced spin precession in $\mathrm{DyFeO}_{3}$, Phys. Rev. B 84, 064402 (2011).
[37] D. Talbayev, J. Lee, S. A. Trugman, C. L. Zhang, S.-W. Cheong, R. D. Averitt, A. J. Taylor, and R. P. Prasankumar, Spin-dependent polaron formation dynamics in $\mathrm{Eu}_{0.75} \mathrm{Y}_{0.25} \mathrm{MnO}_{3}$ probed by femtosecond pump-probe spectroscopy, Phys. Rev. B 91, 064420 (2015).

[38] Y. M. Sheu, Y. M. Chang, C. P. Chang, Y. H. Li, K. R. Babu, G. Y. Guo, T. Kurumaji, and Y. Tokura, Picosecond Creation of Switchable Optomagnets from a Polar Antiferromagnet with Giant Photoinduced Kerr Rotations, Phys. Rev. X 9, 031038 (2019).

[39] G. Gallot and D. Grischkowsky, Electro-optic detection of terahertz radiation, J. Opt. Soc. Am. B 16, 1204 (1999).

[40] R. V. Mikhaylovskiy, T. J. Huisman, A. I. Popov, A. K. Zvezdin, Th. Rasing, R. V. Pisarev, and A. V. Kimel, Terahertz magnetization dynamics induced by femtosecond resonant pumping of $\mathrm{Dy}^{3+}$ subsystem in the multisublattice antiferromagnet $\mathrm{DyFeO}_{3}$, Phys. Rev. B 92, 094437 (2015).

[41] T. J. Huisman, R. V. Mikhaylovskiy, A. Tsukamoto, Th. Rasing, and A. V. Kimel, Simultaneous measurements of terahertz emission and magneto-optical Kerr effect for resolving ultrafast laser-induced demagnetization dynamics, Phys. Rev. B 92, 104419 (2015).

[42] V. V. Eremenko, N. F. Kharchenko, Y. G. Litvinenko, and V. M. Naumenko, in Magneto-Optics and Spectroscopy of Antiferromagnets (Springer-Verlag New York, 1992), p. 276.

[43] V. A. Gavrichkov, S. I. Polukeev, and S. G. Ovchinnikov, Contribution from optically excited many-electron states to the superexchange interaction in Mott-Hubbard insulators, Phys. Rev. B 95, 144424 (2017).

[44] S. G. Ovchinnikov and V. V. Rudenko, Anisotropic interactions in magnetic crystals with S-state ions, Nanostructures. Phys. Usp. 57, 1180 (2014).

[45] See Supplemental Material at http://link.aps.org/ supplemental/10.1103/PhysRevLett.125.157201 for a detailed discussion of our theoretical model and its implications.

[46] V. A. Gavrichkov, S. I. Polukeev, and S. G. Ovchinnikov, Cation spin and superexchange interaction in oxide materials below and above spin crossover under high pressure, Phys. Rev. B 101, 094409 (2020).

[47] R. Diehl, Crystal structure refinement of ferric borate, $\mathrm{FeBO}_{3}$, Solid State Commun. 17, 743 (1975).

Correction: A production processing flaw rendered the affiliation indicators incorrectly for the first and seventh authors in the HTML online version. These affiliation indicators have been fixed and were set properly, without incident, in the PDF version. 